\title{
Comparative numerical study of efficiency of energy deposition in femtosecond microfabrication with fundamental and second harmonics of $\mathrm{Yb}$-doped fiber laser
}

\author{
Alexandr Dostovalov ${ }^{a}$, Sergey Babin ${ }^{a}$, \\ Mandana Baregheh ${ }^{b}$, Mykhaylo Dubov ${ }^{b}$, and Vladimir Mezentzev ${ }^{b}$. \\ ${ }^{a}$ Institute of Automation and Electrometry Siberian Branch of Russian Academy \\ of Sciences, 1 Koptjug Ave, Novosibirsk, 630090 Russia \\ ${ }^{b}$ Photonics Research Group, Aston University, Birmingham B47ET, United Kingdom;
}

\begin{abstract}
We present the results of comparative numerical study of energy deposition in single shot femtosecond laser inscription for fundamental and second harmonic of Yb-doped fiber laser. We have found that second harmonic is more efficient in absorbing energy which leads to lower inscription threshold. Hence this regime is more attractive for applications in femtosecond laser microfabrication
\end{abstract}

\section{INTRODUCTION}

Femtosecond (fs) laser microfabrication is a novel powerful technology in photonics emerged over the last decade. It is geometrically versatile due to 3D flexibility in focusing laser beam under the sample surface. Successfull fs inscription of sculptured microstructures have been recently demonstrated such as low loss waveguides, gratings, couplers, etc. [1-4]. Typically, solid state Ti:Sa fs lasers operating at $800 \mathrm{~nm}$ are exploited for direct inscription in low- and high repetition rate regimes by focusing the laser beam under the sample surface with micro-objectives. The inscription regimes are usually determined by the pulse energy, focusing conditions, and sample translation speed. Operating wavelength is usually fixed or tuned within limited range in the laser setup. However it can be seen as important additional parameter since it determines the order of Multi-Photon Absorption (MPA) which kicks off the process of energy deposition in the inscription domain. Using harmonics of fs laser source is the most straightforward solution for radical change in laser frequency leading to different orders of MPA. There is a few demonstrations of femtosecond inscription exploiting this approach using second [5] and third [6] harmonic of Ti:sapphire femtosecond laser source. However the comparative numerical studies of femtosecond inscription with different harmonics in the same material is absent. Although most of these demonstrations are produced using solid state fs lasers, fiber fs and sub-picosecond fiber lasers are very attractive alternatives for fs microfabrication due to their turn key robustness and efficiency. In this paper we present the results of numerical modelling of energy deposition for typical regimes of femtosecond inscription by fundamental harmonic of an Ytterbium-doped fiber laser at $1030 \mathrm{~nm}$ and its second harmonic at $515 \mathrm{~nm}$. Apart from different number of photons taking part in MPA, shorter laser wavelength of harmonics is also attractive from inscription operation standpoint since it allows for finer resolution of the inscription process.

\section{THEORETICAL MODEL}

Numerical model comprises extended nonlinear Schrödinger equation including effects of diffraction, dispersion, Kerr nonlinearity, multi-photon absorption, plasma absorption and defocusing. All the modelling was performed for laser pulses with fixed FWHM pulsewidth of 300 fs focused with an objective of $N A=0.4$ routinely used in fs inscription experiments. We scanned inscribing pulse energy in the range between few nJ and few hundred $\mathrm{nJ}$ in both cases. It is shown that inscription with second harmonic is generally more efficient than with the fundamental harmonic in terms of energy absorption. Absorbed energy is several times, up to an order of magnitude higher in case of second harmonic which should substantially decrease the inscription threshold.

Further author information: (Send correspondence to A.Dostovalov) e-mail: dostovalov@iae.nsk.su

Fiber Lasers VIII: Technology, Systems, and Applications, edited by Jay W. Dawson, Eric C. Honea, Proc. of SPIE Vol. 7914, $791432 \cdot$ C 2011 SPIE · CCC code: 0277-786X/11/\$18 · doi: 10.1117/12.877631 


\subsection{Equations}

The theoretical model used for calculation of femtosecond pulse propagation in dielectrics and plasma generation is presented in this section. Focused femtosecond pulse initiates plasma generation by multi-photon and subsequent avalanche ionisation. Energy absorption is accepted to be determined by multi-photon and plasma absorption [7]. So the theoretical model must take into account the interaction of these phenomena. We use approach originally suggested by Feit and Fleck [8], and than has been extensively studied [9]:

$$
\begin{gathered}
i \mathcal{E}_{z}+\frac{1}{2 k} \Delta_{\perp} \mathcal{E}-\frac{k^{\prime \prime}}{2} \frac{\partial^{2} \mathcal{E}}{\partial t^{2}}+k_{0} n_{2}|\mathcal{E}|^{2} \mathcal{E}=-\frac{i \sigma}{2}(1+i \omega \tau) \rho \mathcal{E}-i \frac{\beta^{(K)}}{2}|\mathcal{E}|^{2(K-1)} \mathcal{E} \\
\frac{\partial \rho}{\partial t}=\frac{1}{n_{b}^{2}} \frac{\sigma_{b s}}{E_{g}} \rho|\mathcal{E}|^{2}+\frac{\beta^{(K)}}{K \hbar \omega}|\mathcal{E}|^{2 K}
\end{gathered}
$$

The last three terms on the left-hand side of Eq.(1) describe effects of beam diffraction, group velocity dispersion (GVD), and Kerr nonlinerity. The terms on the right-hand side of Eq.(1) are responsible for a pulse energy absorption, namely plasma absorption and multi-photon absorption. In Eq.(1) the laser beam propagation along the $z$ axis is assumed and this equation is essentially a reduced paraxial approximation of the wave equation for the complex electric field envelope $\mathcal{E}$ with a carrier frequency $\omega$ in the moving frame of coordinates. Here $k=n_{b} k_{0}=n_{b} \omega / c$ is the propagation vector, $k^{\prime \prime}=\partial^{2} k(\omega) / \partial \omega^{2}$ is the GVD parameter, $n_{b}(\omega)$ is a linear refractive index of the bulk medium, $n_{2}$ is the nonlinear coefficient describing Kerr effect such that $n_{2}|\mathcal{E}|^{2}$ is a nonlinear contribution to the refractive index, $\sigma_{b s}$ is the cross section for inverse Bremsstrahlung, $\tau$ is the electron relaxation time, $E_{g}$ is the ionization energy, and the quantity $\beta^{(K)}$ describes the $K$-photon absorption. Equation (2) describes the evolution of the electron density $\rho$ in according with the Drude model for electron-hole plasma in the bulk of silica. The first term on the right-hand side is responsible for the avalanche impact ionization and the second term — for the ionization resulting from MPA. It is supposed that plasma recombination and diffusion is negligible. Here, the wave equation describing the evolution of the focused optical beam in the form of NLSE (left-hand terms in Eq.(1)) which is extended to include plasma generation, pulse-plasma interaction, and MPA (terms on the right-hand of Eq.(1). Group velocity dispersion included in Eq.(1) has been shown to lead to pulse splitting and to arrest the collapse $[10,11]$.

\subsection{Physical parameters}

This section describes a set of typical physical parameters for numeric calculation. We assumed the Gaussian initial condition:

$$
\mathcal{E}(z=0, r, t)=\sqrt{\frac{2 P_{i n}}{\pi r_{0}^{2}}} \exp \left(-\frac{r^{2}}{r_{0}^{2}}-\frac{i k r^{2}}{2 f}-\frac{t^{2}}{t_{p}^{2}}\right),
$$

where $r_{0}$ is the waist of the incident beam, $t_{p}$ defines the conventionally defined pulsewidth $t_{F W H M}=\sqrt{2 \ln 2} t_{p} \approx$ $1.177 t_{p}$, and $f$ is a focal length of the objective lens, $P_{i n}$ is initial beam power. Fixed values of $r_{0}=2.5 \mathrm{~mm}$ and $t_{p}=250 \mathrm{fs}$ was used that is according with a radius of microobjective and pulse wight of fs laser source. We assume the ionization energy $E_{g}=9 \mathrm{eV}$, so the order of multiphoton process $K=8$ for the fundamental wavelenght and $K=4$ for the second harmonic. The electron relaxation time $\tau=1.7 \mathrm{fs}$.

As we conducted comparative calculation for fundamental $(1030 \mathrm{~nm})$ and second harmonic $(515 \mathrm{~nm})$ the following parameters are different in both case. Refractive index of fused silica $n_{b}(\omega)$ was calculated using the Sellmeier equation [12]. There are other parameters of material depending on wavelength: GVD coefficient $k^{\prime \prime}=189 \mathrm{fs}^{2} / \mathrm{cm}$ (for fundamental wavelength) and $686 \mathrm{fs}^{2} / \mathrm{cm}$ (for second harmonic), coefficient of multiphoton absorption $\beta^{(8)}=1.2 \times 10^{-117} \mathrm{~cm}^{13} / \mathrm{W}^{7}$ (for fundamental wavelength) and $\beta^{(4)}=1.2 \times 10^{-47} \mathrm{~cm}^{5} / \mathrm{W}^{3}($ for second harmonic), the cross section for inverse Bremsstrahlung $\sigma_{b s}=k_{0} \omega \tau / \rho_{B D}\left(1+\omega^{2} \tau^{2}\right)$.

The condition $f_{e}=f_{p}$, where $f_{e}$ the laser frequency and plasma frequency $f_{p}$, defines the critical (breakdown) density of plasma $\rho_{B D}=\varepsilon_{0} m_{e} e^{-2} \omega^{2}$. This is a resonant condition for conversion of electromagnetic wave into plasma waves. It defines a breakdown electron density $\rho_{B D}$ used below for a given laser frequency, so $\rho_{B D}=1.05 \times 10^{21}$ (for fundamental wavelength) and $\rho_{B D}=4.21 \times 10^{21}$ (for second harmonic) 
The Equation (2) can be expressed as

$$
\frac{\partial}{\partial t} \frac{\rho}{\rho_{B D}}=\frac{1}{n_{b}^{2}} \frac{\sigma_{b s}}{E_{g}} \frac{\rho}{\rho_{B D}}|\mathcal{E}|^{2}+\left(\frac{|\mathcal{E}|^{2}}{I_{M P A}}\right)^{K}
$$

where

$$
I_{M P A}=\left(\frac{K \hbar \omega \rho_{B D}}{\beta^{(K)}}\right)^{1 / K}
$$

is defined to be an MPA threshold as the plasma ionization rate becomes very steep when the intensity $I$ exceeds $I_{M P A}$. Being important physical thresholds, both $I_{M P A}$ and $\rho_{B D}$ were used in a very useful normalization of physical variables to dimensionless ones for performing simulations.

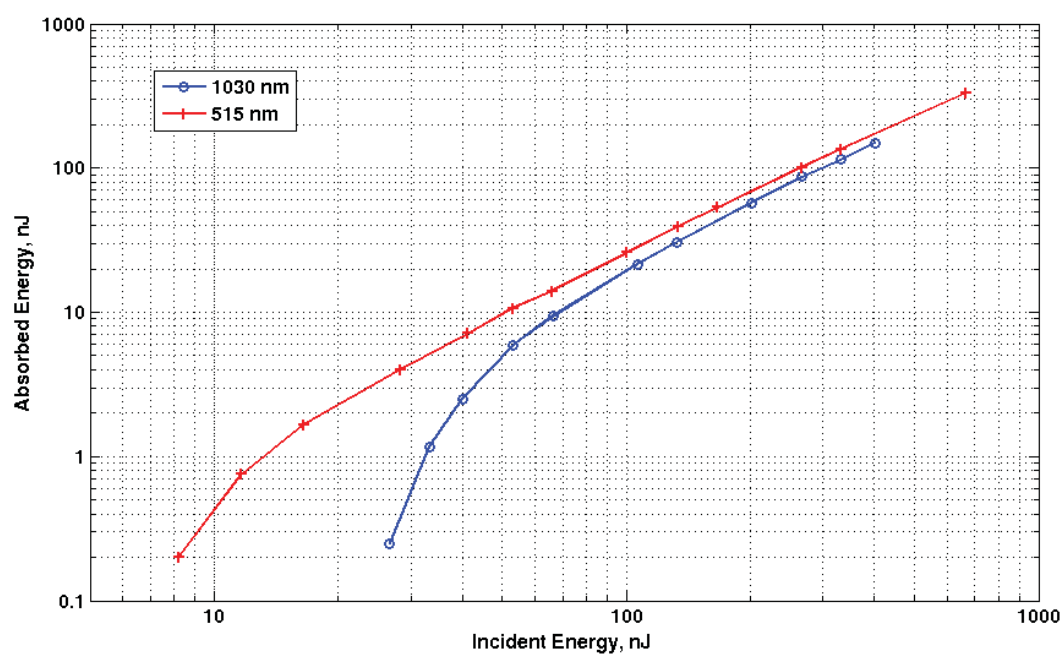

Figure 1. Dependence of absorbed energy upon incident energy. (Logarithmic scale; blue circle - fundamental wavelength, red cross - second harmonic)

\section{NUMERICAL RESULTS AND INTERPRETATION}

Adaptive mesh approach [13] is used for numerical modeling in accord with Eqs(1,2). Nonlinear effects such as self-focusing, multiphoton absorption (MPA), and group velocity dispersion (GVD) are considered in theoretical model. Self focusing leads to beam collapse which means a formation of singularity and on-axis intensity achivies an infinitely high value in finite propogation length [14]. However processes of energy absorption and dispersion prevent the beam collpse. [15]. Application of this method to the Eqs (1,2) was described in our earlier work [13,15] The focused spot is often required to be as small as possible in the microfabrication and the absorbed energy is needed to be within a narrow window between the thresholds of inscription and damage. The dependences of absorbed energy upon incident energy for fundamental and second harmonics in double logarithmic coordinates are presented in Fig. 1. Different behavior is seen for low and higher energies around $40 \mathrm{~nJ}$. It is natural to assume that different mechanisms are responsible for absorption in these regions. It is considered that MPA process dominates in the low energy region, while the plasma absorption prevails for higher energies, so the dependence of absorption tends to be linear [16]. It is shown that adsorbed energy for second harmonic is higher than for fundamental wavelenght at fixed incident energy. In this reason, second harmonic is more preferable for fs inscription because the inscription threshold is lower in this case.

The adaptive procedure [13] allows for accurate treatment of multiscale (time and space) evolution which results in stationary (in the framework of the model considered) distribution of plasma. The mechanisms of eventual plasma recombination and subsequent relaxation of the medium are extremely complex. Accurate spatial distribution of 
plasma is necessary because the eventual material modification depends on plasma distrubution. The stationary spatial distribution of electrons can be found as

$$
\rho(\mathbf{r}, z)=\int_{-\infty}^{\infty}\left[\frac{1}{n_{b}^{2}} \frac{\sigma}{E_{g}} \rho|\mathcal{E}(\mathbf{r}, z, t)|^{2}+\frac{\beta^{(K)}}{K \hbar \omega}|\mathcal{E}(\mathbf{r}, z, t)|^{2 K}\right] d t .
$$

Density plots of plasma concentrations are shown in one transverse and one propagation coordinates. The stationary plasma profiles at $\lambda=515 \mathrm{~nm}$ and different initial pulse energies (23 nJ, $66 \mathrm{~nJ}, 165 \mathrm{~nJ})$ are presented in Fig. 2. The plasma distrubution for the lower pulse energy ( $23 \mathrm{~nJ}$ ) is centrosymmetrical (using chosen scale), while plasma distribution for the higher pulse energy $(165 \mathrm{~nJ})$ has comlex shape. There are the wide initial part and pointed part of plasma distrubution shape. The stationary plasma profiles for fundamental and second harmonics with fixed energy $(66 \mathrm{~nJ})$ are shown in Fig. 3. It shows that plasma distribution at $\lambda=515 \mathrm{~nm}$ has the finer arrowy area than the shape of plasma distribution at $\lambda=1030 \mathrm{~nm}$. So it is more preferable to utilize $\lambda=$ $515 \mathrm{~nm}$ for microfibrication in particular for producing of first-order fiber Bragg gratings. While the inscription regime at $\lambda=1030 \mathrm{~nm}$ is the most attractive for microfabrication of large scale structures such as waveguides because there is smooth plasma distribution that leads to smooth refractive index change. Collapse of the laser beam due to self-focusing is eventually arrested by multiphoton absorption resulting in a very confined plasma cloud.

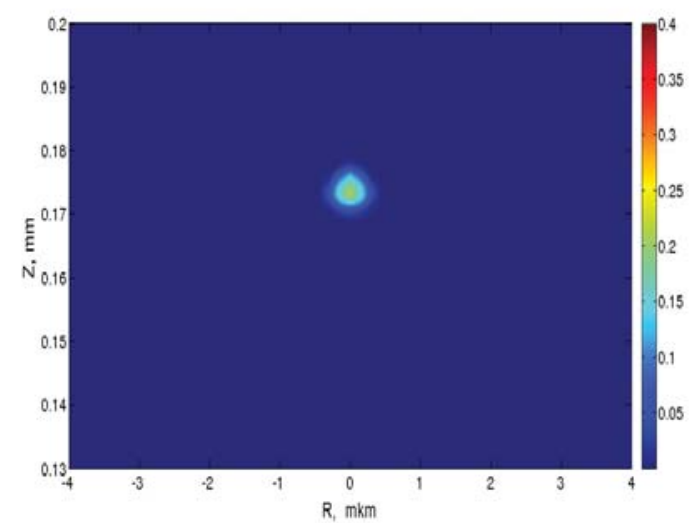

a)

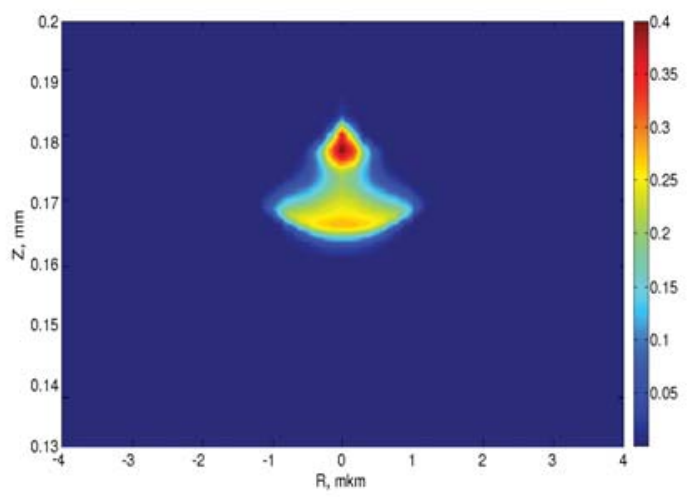

c)

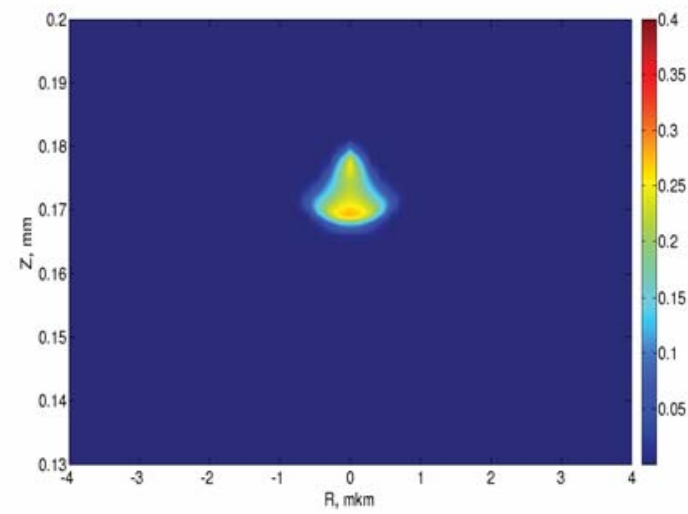

b)

Figure 2. Plasma density distribution for fixed wavelength $\lambda=515 \mathrm{~nm}$ and different initial pulse energies: a) $23 \mathrm{~nJ}$, b) $66 \mathrm{~nJ}, \mathrm{c)} 165 \mathrm{~nJ}$. 

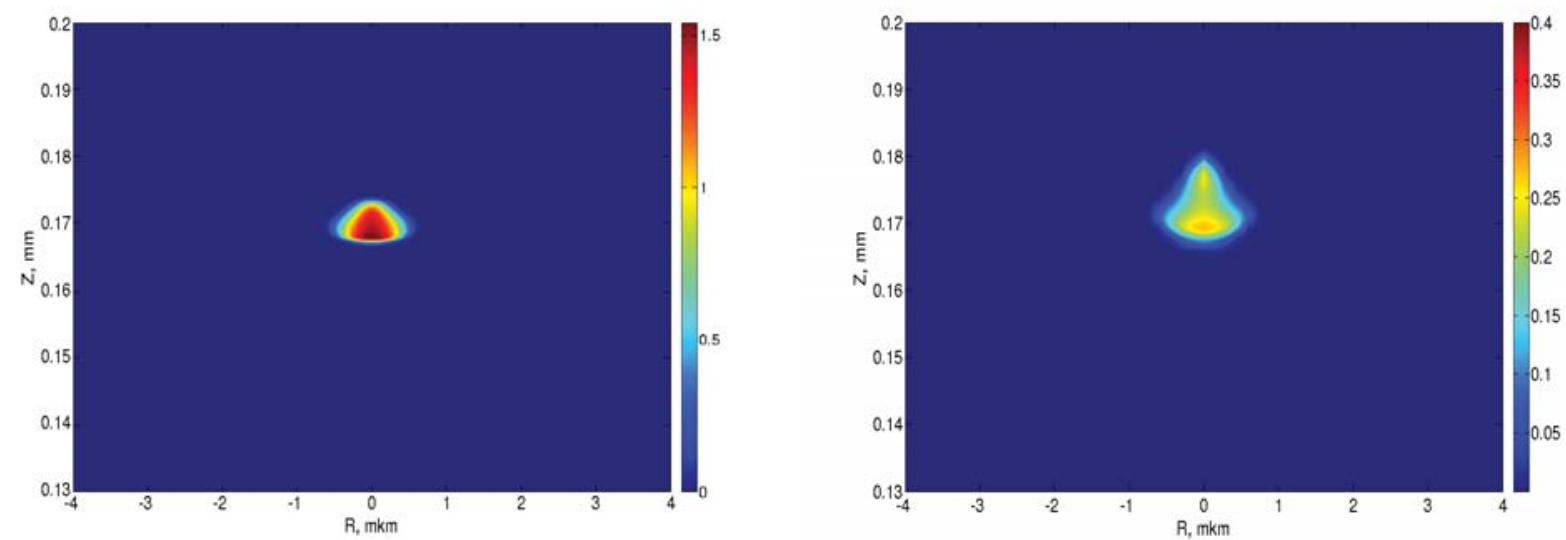

Figure 3. Plasma density distribution for fixed pulse energy $(66 \mathrm{~nJ})$ and different wavelengths: $\lambda=1030 \mathrm{~nm}(\mathrm{left}), \lambda=$ $515 \mathrm{~nm}$ (right).

\section{CONCLUSION}

The results of comparative numerical modeling of energy deposition of femtosecond pulse for fundamental and second harmonics of $\mathrm{Yb}$-doped fiber laser are presented. It is shown that utilizing of second harmonic for fs inscription is more preferable, because the inscription threshold is lower in this case. And the area of refractive index modification is finer than for fundamental wavelength. Also the evolution of plasma density distribution for second harmonic upon initial pulse energy are presented. That reveals the pointed area at higher energy so this regime is preferable for achieving the finer sizes of fs modification in particular for producing of fiber Bragg gratings.

\section{ACKNOWLEDGMENTS}

The authors acknowledge support from the Russian Ministry of Education and Science (grant no. 02.740.11.5063) and integration project of Siberian Branch of Russian Academy of Science.

\section{REFERENCES}

[1] R. R. Gattass, E. Mazur, "Femtosecond laser micromachining in transparent materials" Nature 2, pp. 219$225,2008$.

[2] M. Dubov, I. Bennion, "Strong long-period fiber gratings recorded at 352nm" Opt. Lett. 19, pp. 2533-2535, 2005.

[3] M. Dubov, V. Mezentsev, I. Bennion, D. N. Nikogosyan, "UV femtosecond laser inscribes a $300 \mathrm{~nm}$ period nanostructure in a pure fused silica" Meas.Sci.Technol. 18, pp. 219-225, 2007.

[4] G. N. Smith, K. Kalli, K. Sugden, "Advances in femtosecond micromachining and inscription of micro and nano photonic devices" Frontiers Guided Wave Optics and Optoelectrinics 15, pp. 295-320, 2010.

[5] K. A. Zagorulko, P. G. Kryukov, Y. V. Larionov, A. A. Rybaltovsky, E. M. Dianov, N. S. Vorobev, A. V. Smirnov, M. Y Schelev, A. M. Prokhorov. "Fabrication of a long-period grating in a fibre by second-harmonic radiation from a femtosecond Ti:sapphire laser" Quantum Electron 31, 2001.

[6] M. Dubov, I. Bennion, D. N. Nikogosyan, P. Bolger, A. V. Zayats "Point-by-point inscription of $250 \mathrm{~nm}$ period structure in bulk fused silica by tightly focused femtosecond UV pulses" J. Opt. A:Pure Appl. Opt. 10, pp. 1-6, 2008.

[7] A. G. Okhrimchuk V. K. Mezentsev, H. Schmitz, M. Dubov, and I. Bennion, "Cascaded nonlinear absorption of femtosecond laser pulses in dielectrics" Laser Physics 7, pp. 1415-1422, 2009.

[8] M. D. Feit and J. A. Fleck, "Effect of refraction on spot-size dependence of laser-induced breakdown," Appl. Phys. Lett. 24, pp. 169-172, 1974. 
[9] L. Berge, S. Skupin, R. Nuter, J. Kasparian, J. P. Wolf, "Ultrashort filaments of light in weakly ionezed, optically transparent media," Rep. Prog. Phys 70, pp. 1633-1713, 2007.

[10] P. Chernev and V. Petrov, "Self-focusing of light pulses in the presence of normal group velocity dispersion," Opt. Lett. 17, pp. 172-174, 1992.

[11] J. E. Rothenberg, "Pulse splitting during self-focusing in normally dispersive media," Opt. Lett. 17, pp. 583585, 1992.

[12] G. P. Agrawal, [Nonlinear fiber optics] San Diego, CA: Academic Press, 1995.

[13] V. Mezentsev, J. Petrovic, J. Dreher, and R. Grauer, "Adaptive modeling of the femtosecond inscription in silica," in Laser-based Micropackaging, F. G. Bachmann, W. Hoving, Y. Lu, and K. Washio, eds., Proc. SPIE 6107, p. 61070R, 2006.

[14] S. Vlasov, V. Petrishchev, and V. Talanov Izv. Vuzov, Radiofizica 14, pp. 1353-1364, 1971.

[15] K. Germaschewski, R. Grauer, L. Bergé, V. K. Mezentsev, and J. J. Rasmussen, "Splittings, coalescence, bunch and snake patterns in the $3 \mathrm{~d}$ nonlinear schrodinger equation with anisotropic dispersion," Physica D 151, pp. 175-198, 2001.

[16] M. J. Berger and P. Colella, "Local adaptive mesh refinement for shock hydrodynamics," J. Comp. Phys. 82, pp. 64-84, 1989. 